\title{
NOTE ON REGIONS OMITTED BY UNIVALENT FUNCTIONS
}

A. W. GOODMAN

1. Introduction. Let $\mathfrak{F}$ denote the set of functions

$$
w=f(z)=\sum_{n=1}^{\infty} a_{n} z^{n}, \quad\left|a_{1}\right|=1,
$$

which are regular and univalent in $E$, the open unit circle. A classic result due to Koebe ${ }^{1}$ states that if $f(z) \in \mathscr{F}$ omits $\nu$ for $z$ in $E$, then $|\nu| \geqq 1 / 4$, and that equality occurs (essentially) ${ }^{2}$ only for the function

$$
k(z)=\frac{z}{(1+z)^{2}} .
$$

Hence the problem for a single omitted value is completely solved.

It is natural to inquire into the situation when the set of omitted values contains a region or a number of regions. This can be specialized in two ways. In $\$ 2$ it is assumed that $f(z)$ omits all the values inside a circle of radius $R$, and a sharp upper bound for $R$ is obtained. In $\S 3$ the area of the intersection of $E$ and the set of omitted values is considered. The result obtained is not sharp.

2. The largest circle of omitted values. We shall make use of the following theorem due to Pick $^{3}$ and Nevanlinna, ${ }^{4}$ and since a short proof can be given, it is included here for completeness.

Theorem 1. Let $h(z) \in \mathfrak{F}$ omit $\gamma$ in E. Further suppose that $|h(z)|$ $<M$ for $z$ in $E$. Then

$$
|\gamma| \geqq 2 M^{2}-M-2 M\left(M^{2}-M\right)^{1 / 2},
$$

with equality (essentially) only for the function

$$
h(z)=M K\left[\frac{1}{M} k(z)\right]
$$

Received by the editors January 24, 1948, and, in revised form, April 14, 1948.

1 Titchmarsh, The theory of functions, Oxford University Press, p. 211.

2 We use "(essentially)" to denote that all other functions for which the equality sign holds can be obtained from the one given by subjecting both planes to appropriate rotations.

${ }^{3}$ G. Pick, Sitzungsberichte der Kaiserlichen Akademie der Wissenschaften, Vienna, Abteilung IIa, vol. 126 (1917) pp. 247-263.

4. Revanlinna, Oversigt a Finska Vetenskaps Societetens Forhandlinger vol. 62 (1919). 
where $K(z)$ denotes the inverse function of $k(z)$.

Proof. Without loss of generality take $\gamma>0$. Clearly

$$
f(z)=\frac{h(z)}{(1+h(z) / M)^{2}}
$$

is in $\mathfrak{F}$ and omits the positive value

$$
\nu=\frac{\gamma}{(1+\gamma / M)^{2}} .
$$

Apply Koebe's Theorem. Since, for fixed $M,(6)$ is an increasing func-

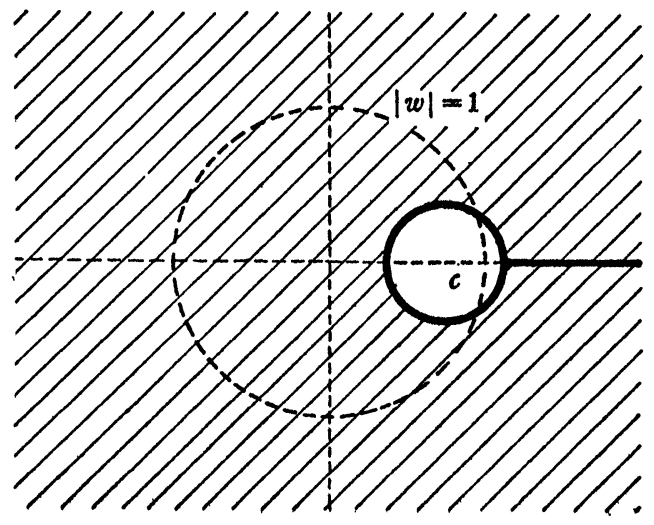

Fig. 1

tion of $\gamma$ in the interval $0 \leqq \gamma \leqq M$, the inequality $\nu \geqq 1 / 4$ implies $\gamma \geqq \gamma_{c}$, the least positive root obtained by solving

$$
\frac{\gamma}{(1+\gamma / M)^{2}}=\frac{1}{4} \text {. }
$$

This yields for $\gamma_{c}$ exactly the right side of (3). Since $k(z)$ is (essentially) the only function for which $\nu=1 / 4$, it is easy to see that (4) is (essentially) the only function for which the equality sign holds in (3). The function given by (4) maps $E$ onto a circle of radius $M$ with a slit along the positive real axis from $M$ to $\gamma$.

THEOREM 2. Let $f(z) \in \mathfrak{F}$, Let $c$ be fixed and suppose that for $z$ in $E$, $f(z)$ omits all $\xi$ for which

$$
|\xi-c| \leqq R
$$

Then 


$$
R \leqq|c| \frac{4|c|-1}{4|c|+1}
$$

with equality (essentially) only for the function which maps $E$ onto the entire complex plane except for the closed circle of prescribed center and radius, and a radial slit running from the point $c+(c /|c|) R$ to the point at infinity (see Fig. 1).

Proof. Without loss of generality take $f^{\prime}(0)=1$ and $c>0$. Since $f(0)=0, c-R>0$. Let

$$
g(z)=\frac{R}{f(-z)-c} .
$$

Then for $z$ in $E, g(z)$ is regular, univalent, $|g(z)|<1$, and $g(z) \neq 0$. Also $g(0)=-R / c$, and $g^{\prime}(0)=R / c^{2}$. Next set

$$
h(z)=\frac{\left(c^{2}-R^{2}\right)(g(z)-g(0))}{R(1-g(z) g(0))} .
$$

Then $h(z)$ satisfies the conditions of Theorem 1 with

$$
M=\left(c^{2}-R^{2}\right) / R, \quad \gamma=\left(c^{2}-R^{2}\right) / c>0 .
$$

We write (3) in the form

$$
2 M\left(M^{2}-M\right)^{1 / 2} \geqq 2 M^{2}-(M+\gamma) .
$$

Now $M>1$ and $M>\gamma$ show that the right side of (13) is positive, and so we may square both sides and obtain

$$
4 M^{2} \gamma \geqq(M+\gamma)^{2} .
$$

Using (12) in (14) and simplifying, one finds

$$
4 c(c-R) \geqq R+c,
$$

and this is equivalent to (9).

To see that the inequality is sharp and to obtain the (essentially) unique function, reverse the steps of the proof. Take $h(z)$ as given by (4) with $M$ determined by (12) and $R$ determined by (9) with the equality sign. Then (11) together with the requirement that $g(0)$ $=-R / c$ gives $g(z)$. Finally (10) defines $f(z)$, and it is not difficult to verify that the $f(z)$ so obtained has all of the required properties.

Theorem 3. Let $h(z) \in \mathfrak{F}$ omit $\gamma$ in $E$. Further suppose that in $E$, $\Re(h(z))>-c, c>0$. Then

$$
|\gamma| \geqq c-\left(4 c^{2}-2 c\right)^{1 / 2} / 2
$$


with equality only in the case $h(z)$ maps $E$ onto the half-plane $\Re(\omega)>-c$, slit along the real axis from $-c$ to $-c+\left(4 c^{2}-2 c\right)^{1 / 2} / 2$.

The proof of this theorem is similar to that of Theorem 2, and will be omitted.

3. The area question. Instead of the point set mentioned in $\$ 1$, it will be more convenient and completely equivalent to consider the set $S_{f}$, the intersection of $E$ and the set of values assumed by $f(z)$ for $z$ in $E$. Let $A_{f}$ be the area of this set and let $A$ denote the greatest lower bound of $\left\{A_{f}\right\}$ for $f(z) \in \mathfrak{F}$. Roughly speaking, $A$ measures the amount of the unit circle which must always remain covered by the map of $E$ under any normalized univalent function. We shall prove the following theorem.

THEOREM 4.

$$
.5000 \pi \leqq A<.7728 \pi \text {. }
$$

In establishing the lower bound use will be made of the rather elementary lemma:

Lemma 1. Let $k>0$. Let $\phi(\theta)$ be a positive continuous function for $0 \leqq \theta \leqq 2 \pi$, and

$$
\int_{0}^{2 \pi} \phi(\theta) d \theta \leqq c
$$

Then

$$
\int_{0}^{2 \pi} \frac{d \theta}{\phi^{k}(\theta)} \geqq \frac{(2 \pi)^{k+1}}{c^{k}}
$$

with equality if and only if $\phi(\theta) \equiv c / 2 \pi$.

The Hölder inequality for integrals ${ }^{5}$ states that if $\alpha, \beta, f(x), g(x)$ are positive and $\alpha+\beta=1$, then

$$
\int_{0}^{2 \pi} f^{\alpha}(\theta) g^{\beta}(\theta) d \theta \leqq\left[\int_{0}^{2 \pi} f(\theta) d \theta\right]^{\alpha}\left[\int_{0}^{2 \pi} g(\theta) d \theta\right]^{\beta} .
$$

Take $\alpha=1 / k+1, \beta=k / k+1, g(\theta)=\phi(\theta), f(\theta)=(\phi(\theta))^{-\beta / \alpha}=\phi^{-k}(\theta)$, and obtain

$$
2 \pi \leqq\left[\int_{0}^{2 \pi} \frac{d \theta}{\phi^{k}(\theta)}\right]^{1 /(k+1)} c^{k /(k+1)} .
$$

\footnotetext{
5 Hardy, Littlewood, and Pólya, Inequalities, Cambridge University Press, p. 140.
} 
This gives (18). Since equality occurs in (19) only when $f(\theta)$ and $g(\theta)$ are proportional, equality occurs in (20) only if $\phi(\theta)$ is constant.

To establish the lower bound for $A$ consider, not $f(z)$, but $f(r z) / r$ $=f_{r}(z), 0<r<1$. This insures the regularity of $f_{r}(z)$ on the boundary of $E$. To simplify matters, assume that the boundary of $S_{f_{r}}$ is given

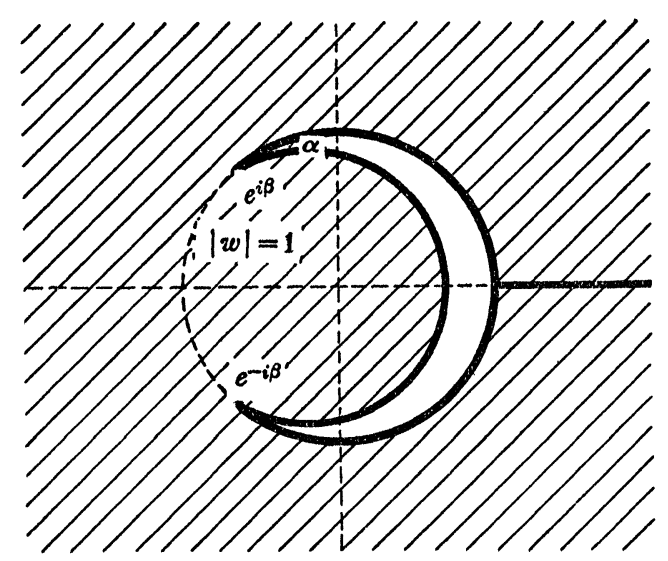

Fig. 2

by $R=\phi_{r}(\theta)$, a continuous single-valued function for $0 \leqq \theta \leqq 2 \pi$. Then

$$
A_{f_{r}}=\frac{1}{2} \int_{0}^{2 \pi} \phi_{r}^{2}(\theta) d \theta .
$$

Hence the set of points omitted by $1 / f_{r}(z)$, which lie outside the unit circle, has area

$$
Q=\frac{1}{2} \int_{0}^{2 \pi} \frac{d \theta}{\phi_{r}^{2}(\theta)}-\pi .
$$

But by the Faber-Bieberbach ${ }^{6}$ area theorem $Q \leqq \pi$ and so

$$
\int_{0}^{2 \pi} \frac{d \theta}{\phi_{r}^{2}(\theta)} \leqq 4 \pi
$$

By Lemma 1, with $k=1$

$$
2 A_{f_{r}}=\int_{0}^{2 \pi} \phi_{r}^{2}(\theta) d \theta \geqq \frac{(2 \pi)^{2}}{4 \pi}=\pi .
$$

It is easy to remove the restriction that $\phi_{r}(\theta)$ be single-valued and continuous. The details will be omitted, but it should be noted that

- Titchmarsh, loc. cit. page 209. 
for the functions $f_{r}(z)$ each ray through the origin intersects the boundary of $S_{f_{r}}$ in at most a finite number of points. Finally taking the limit as $r \rightarrow 1$ presents no difficulties.

To establish the upper bound we construct, by combining the five simple functions (25), a function $w=f(z)$ which maps $E$ onto the shaded region shown in Fig. 2.

$$
\begin{array}{rlrl}
\eta & =K[c k(z)], & 0<c<1, \\
\zeta & =\frac{\eta+\zeta_{0}}{\zeta_{0} \eta+1}, & -1<\zeta_{0}<0, \\
u & =\frac{1+i \zeta}{1-i \zeta}, & \\
v & =u^{2-\alpha / \pi}, & & 0 \leqq \alpha<\pi, \\
w & =\frac{e^{i \beta v}+e^{i(\pi-\alpha / 2)}}{v+e^{i(\pi-\alpha / 2+\beta)}}, & 0<\beta<\pi .
\end{array}
$$

The first mapping takes $E$ into $E$ with a slit with length depending on the parameter $c$. The second preserves $E$ and introduces a parameter $\zeta_{0}$ which is later determined by the condition $f(0)=0$. The third mapping takes $E$ into the right half-plane, and the fourth takes this half-plane into the entire plane except for a wedge of angular aperture $\alpha$ symmetrically located about the negative real axis with apex at the origin. The slit in $E$ becomes a circular arc slit of unit radius and one end on the upper boundary of the wedge. Finally the fifth mapping takes this region onto the shaded region of Fig. 2.

The constant $c$ is determined by requiring that the slit end go into the point at infinity. Using subscripts to denote the images of the points in the $w$-plane it is easy to verify the following.

$$
\begin{aligned}
u_{0} & =e^{i \psi_{0},} & \psi_{0} & =\frac{-\pi}{2 \pi-\alpha}\left(\beta+\frac{\alpha}{2}\right), \\
u_{\infty} & =e^{i \psi_{\infty},} & \psi_{\infty} & =\frac{\pi}{2 \pi-\alpha}\left(\beta-\frac{\alpha}{2}\right), \\
\zeta_{0} & =\frac{\sin \psi_{0}}{1+\cos \psi_{0}}, & \zeta_{\infty} & =\frac{\sin \psi_{\infty}}{1+\cos \psi_{\infty}}, \\
\eta_{\infty} & =\frac{\sin \left(\left(\psi_{\infty}-\psi_{0}\right) / 2\right)}{\cos \left(\left(\psi_{\infty}+\psi_{0}\right) / 2\right)}, & c & =4 k\left(\eta_{\infty}\right) .
\end{aligned}
$$


This gives the two parameters $c$ and $\zeta_{0}$ as functions of $\alpha$ and $\beta$. Finally

$$
\left|f^{\prime}(0)\right|=\frac{2 \pi-\alpha}{\pi \sin \beta} 4 k\left(\eta_{\infty}\right) \cos \psi_{0} .
$$

Setting $\left|f^{\prime}(0)\right|=1$ gives a transcendental equation which determines $\alpha$ as a function of $\beta$. Fortunately, however, it is not necessary to carry the precise analysis any further to secure a valid upper bound for $A$. The particular selection $\alpha=28^{\circ}, \beta=90^{\circ}$ gives $\left|f^{\prime}(0)\right|>1.0015$ and $A_{f}<.7728 \pi$.

To place our conclusion on a completely sound basis it must be remarked that by a well known lemma ${ }^{7}\left|f^{\prime}(0)\right|$, for fixed $\beta$, is a strictly decreasing function of $\alpha$. Further $\left|f^{\prime}(0)\right|$ is a continuous function of $\alpha$. So with $\beta=90^{\circ}$ there is a unique $\alpha^{\prime}$ for which $\left|f^{\prime}(0)\right|=1$ and $\alpha^{\prime}>28^{\circ}$.

\section{RUTGERS UNIVERSITY}

7 If $f(z)$ and $g(z)$ map $E$ onto $F$ and $G$ respectively with $f(0)=g(0)$ and $F \subset G$, then $\left|f^{\prime}(0)\right|<\left|g^{\prime}(0)\right|$. 